\title{
Des enseignantes du secondaire dans l'Empire Britannique
}

Identités professionnelles, genre et mission religieuse

Women Secondary School Teachers and Empire : professional identities, gender and religious mission

Über die Sekundarschullehrerinnen im britischen Empire : Berufsbilder, Geschlecht und religiöse Aufgabe

\section{Joyce Goodman}

Traducteur : Céline Grasser

\section{OpenEdition}

Journals

Édition électronique

URL : https://journals.openedition.org/histoire-education/992

DOI : 10.4000/histoire-education.992

ISSN : 2102-5452

Éditeur

ENS Éditions

Édition imprimée

Date de publication : 1 mai 2003

Pagination : 109-132

ISBN : 2-7342-0950-0

ISSN : 0221-6280

\section{Référence électronique}

Joyce Goodman, «Des enseignantes du secondaire dans l'Empire Britannique », Histoire de l'éducation [En ligne], 98| 2003, mis en ligne le 12 mars 2009, consulté le 20 mai 2021. URL : http://

journals.openedition.org/histoire-education/992; DOI : https://doi.org/10.4000/histoire-education.992

Ce document a été généré automatiquement le 20 mai 2021.

(c) Tous droits réservés 


\title{
Des enseignantes du secondaire dans l'Empire Britannique
}

\author{
Identités professionnelles, genre et mission religieuse \\ Women Secondary School Teachers and Empire : professional identities, gender \\ and religious mission \\ Über die Sekundarschullehrerinnen im britischen Empire : Berufsbilder, \\ Geschlecht und religiöse Aufgabe
}

Joyce Goodman

Traduction : Céline Grasser

En 1905, Miss Gertrude Edwards, une enseignante de Godolphin School, institution basée à Salisbury, quitte l'Angleterre pour l'Afrique du Sud afin d'occuper le poste de directrice de St Michael's School à Bloemfontein. Elle est accompagnée de sa sœur célibataire, Adeline, qui avait travaillé avec elle à Godolphin comme enseignante. D'autres membres du personnel et d'anciennes élèves de Godolphin gagnent à leur tour l'Afrique du Sud pour travailler à St Michael's School avec elle. Des ennuis de santé, ou le fait de se marier, provoquent le retour en métropole de certaines qui sont rapidement remplacées. D'autres font le voyage en sens inverse et quittent St Michael's School pour occuper un poste en Angleterre, à Godolphin. Miss Armstrong rejoint ainsi Salisbury «en provenance directe de l'établissement de Miss Edwards à Bloemfontein $»^{1}$. Le personnel de Godolphin intègre également la Diocesan School de Grahamstown en Afrique du Sud. En 1903, Miss Wilsden, une ancienne enseignante de Godolphin ouvre la voie. En 1913, Miss Ethel Jones quitte à son tour Salisbury pour occuper le poste de directrice de la Diocesan School de Grahamstown, où elle « joue un rôle d'aimant ». En quelques semaines, plusieurs femmes de Godolphin la rejoignent: Miss Ethel Young, maîtresse et également ancienne de l'école, ainsi que deux autres anciennes, Ursula Strokes, en qualité d'économe, ou d'intendante, et Joan Shorto, qui vient enseigner les mathématiques².

2 Ces enseignantes du secondaire qui quittent Godolphin School en Angleterre pour rejoindre des postes dans les deux établissements sud-africains mentionnés ne sont pas 
des exceptions. Le désir d'émigrer caractérise les enseignantes les plus motivées ${ }^{3}$, qui font partie d'une diaspora de femmes britanniques éduquées qui n'hésitent pas à franchir les frontières internationales à une époque où l'émigration, dans le cas des femmes seules, est considérée comme peu judicieuse, voire franchement dangereuse ${ }^{4}$. Un grand nombre de celles qui quittent l'Angleterre pour occuper des postes dans des établissements scolaires secondaires outre-mer ont fait leurs études dans des colleges féminins anglais 5 . D'autres sont nées à l'étranger et se sont rendues en Angleterre pour $\mathrm{y}$ faire leurs études, avant de regagner leur pays d'origine, voire de gagner un troisième et parfois un quatrième pays pour enseigner ${ }^{6}$. Le passage par les colleges féminins anglais, où les principales encouragent les étudiantes à occuper des postes d'enseignantes au-delà des frontières, est à l'origine de l'intégration des établissements scolaires d'outre-mer au sein d'un réseau de femmes formées dans l'enseignement supérieur, qui trouvent un soutien dans leurs associations tant professionnelles que d'anciennes élèves? .

3 Cet article explore les relations entre identités professionnelles, vocation à servir, religion, mission, Empire et identité nationale anglaise qui apparaissent dans le cadre de la circulation internationale des enseignantes et directrices de l'enseignement secondaire féminin de la fin des années 1890 à 1914. Sont d'abord examinées les stratégies adoptées pour faciliter le déplacement outre-mer des enseignantes du secondaire par l'Association of Headmistresses (Association des directrices), qui est, en Angleterre, la plus importante des organisations professionnelles d'enseignantes des établissements secondaires pour filles. L'analyse des positions, activités et stratégies de l'Association se fonde sur ses rapports annuels imprimés et sur les procès-verbaux de son comité exécutif et de ses commissions. Une seconde partie s'intéresse plus spécifiquement aux missions religieuse et impériale, une étude de cas menée à partir de matériaux anglais de Godolphin permettant d'explorer la circulation à double-sens existant entre cette institution et les deux établissements sud-africains que sont St Michael's School de Bloemfontein et la Diocesan School de Grahamstown. Cette étude de cas se fonde sur des informations publiées dans le journal scolaire de Godolphin, en particulier sur les lettres et les récits envoyés à Godolphin par celles qui travaillent au sein des deux institutions d'Afrique du Sud, toutes deux fondées en 1874 par des évêques anglais en tant qu'établissements anglicans à fort esprit religieux, à l'usage des jeunes filles sud-africaines blanches ${ }^{8}$.

4 Sont également utilisés des comptes rendus des cérémonies de distribution des prix ainsi que des services de commémoration publiés dans le magazine de l'école et dans les journaux locaux, qui consistent soit en comptes rendus littéraux des discours, soit en descriptions de première main des événements par des élèves ou des membres du personnel. Les discours prononcés lors de la cérémonie de distribution des prix (qui a lieu une fois par an pour la remise de diplômes et de prix aux élèves), de même que les discours et les sermons prononcés le jour de la commémoration annuelle (cérémonie réunissant les élèves de l'établissement et les anciennes, lors de laquelle se voient réaffirmés les fondements et les idéaux de l'école), offrent une vision officielle de la façon dont les différents personnels des établissements conçoivent des relations coloniales qui ont également une dimension sexuée. Les positions du conseil d'administration, qui a officiellement pour responsabilité de surveiller la gestion et l'organisation de la stratégie générale de l'école, revêtent une importance particulière dans ce contexte, tandis que sont également retracés les liens des membres de ce conseil avec l'Empire. La manière dont des conceptions des relations coloniales venues 
d'outre-mer se répandent par le biais de réseaux transnationaux dans le domaine de l'éducation secondaire des filles en Grande-Bretagne même constitue une question-clé de cette partie. Enfin, la dernière partie de cet article souligne l'existence de différentes strates d'appartenance culturelle au niveau des trois établissements scolaires, qui déterminent la position des écoles, de leurs élèves et de leurs personnels les unes par rapport aux autres. Les écarts d'appartenance se reflètent aussi dans les relations différentielles forgées par l'Association of Headmistresses avec les directrices des institutions anglaises et leurs homologues des établissements d'outre-mer.

\section{L'Association of Headmistresses et l'Empire}

5 L'Association of Headmistresses est fondée en 1874 par Frances Mary Buss en tant qu'organisation professionnelle s'adressant aux directrices gérant les nouveaux établissements scolaires secondaires publics pour filles. En 1906, l'effectif est passé des neuf adhérentes initiales à un total de 230 personnes couvrant tout le pays, chiffre qui double à nouveau dès 1912, avec l'admission des directrices des écoles secondaires municipales fondées à la suite de l'Education Act (Loi sur l'Éducation) de $1902^{9}$. L'association organise une rencontre annuelle pour ses membres, met en place un certain nombre de sous-commissions chargées d'enquêter sur des questions éducatives, et publie toute une gamme de brochures et de livres portant sur des sujets liés à l'éducation.

6 C'est à partir de 1897 que l'Association commence à s'intéresser à des questions éducatives concernant l'Empire ${ }^{10}$, avant de développer plus systématiquement ce type d'activités lorsqu'elle noue des liens formels avec la League of Empire et la Victoria League, toutes deux fondées en $1901^{11}$. La League of Empire met en place toute une série de programmes éducatifs destinés à encourager l'unité impériale, parmi lesquels un club de correspondance pour écoliers, des célébrations à l'occasion de l'Empire Day (Jour de l'Empire), des pièces patriotiques à l'usage des clubs de théâtre scolaires et des chants à la gloire de la patrie ${ }^{12}$. La Victoria League for Commonwealth Friendship a été créée dans le but de favoriser la compréhension mutuelle et la mise en place de liens plus étroits entre les populations des Îles britanniques et celles de l'Empire. Elle facilite notamment les échanges d'enseignants entre diverses parties du Commonwealth ${ }^{13}$. L'Association of Headmistresses est représentée officiellement au sein des comités de ces deux sociétés, qui organisent toutes deux des colloques portant spécifiquement sur l'éducation à Londres, le premier de ces colloques destinés aux associations d'enseignants de l'Empire ayant lieu en 1912 sous le patronage de la League of Empire ${ }^{14}$.

7 L'Association of Headmistresses établit sa propre commission coloniale en 1907, ce qui coïncide avec les préparatifs du Pan-Anglican Congress (Congrès pan-anglican) tenu à Londres en 1908. Issu d'une initiative de l'Anglican Central Board of Missions (Comité central anglican des missions), ce congrès s'organise autour de sept thèmes : L'Église et la société humaine, L'Église et la pensée humaine, le sacerdoce, les missions de l'Église en terre non chrétienne, les missions de l'Église au sein de la chrétienté, la communion anglicane, et enfin l'Église et les jeunes. Chaque diocèse d'outre-mer de confession anglicane est invité à envoyer six délégués. On compte parmi les déléguées féminines des missionnaires et des femmes actives au sein de l'Église et de l'assistance sociale outre-mer ${ }^{15}$. Le Comité féminin du Pan-Anglican Congress organise une série de rencontres portant sur des sujets intéressant les femmes et les jeunes filles, ainsi 
qu'une grande assemblée féminine au Albert Hall le dernier soir du congrès ${ }^{16}$. Tout comme la Girls Friendly Society et la Mothers Union, qui programment leurs propres rencontres impériales parallèlement au congrès pan-anglican principal ${ }^{17}$, $l^{\prime}$ Association of Headmistresses tient sa Conférence annuelle la même semaine ${ }^{18}$ et organise une rencontre qualifiée de At Home à Clapham High School pour faire connaissance avec les directrices venues d'outre-mer assister au congrès ${ }^{19}$.

Une branche complémentaire d'actions impériales se développe au sein de l'Association of Headmistresses du fait de la volonté des directrices de fournir des informations à leurs élèves sur les perspectives en matière d'emploi, tant en Angleterre que dans les colonies britanniques, avec pour conséquence la mise en place d'une relation officielle entre l'association et le Committee of Colonial Intelligence for Educated Women (comité des renseignements coloniaux à l'usage des femmes instruites). Ce dernier est fondé en 1910 à l'initiative de membres de la British Women's Emigration Association et de la South African Colonisation Society, qui considèrent qu'une connaissance plus «autorisée » des possibilités d'emploi est nécessaire pour pousser à l'émigration les femmes «instruites" avec «succès ${ }^{20}$. Ce Committee of Colonial Intelligence se reforme sous le nom de Colonial Intelligence League, une moitié des sièges de son comité exécutif étant occupée par des membres de l'Association of Headmistresses, qui s'entendent pour promouvoir l'action de la ligue dans leurs établissements scolaires ${ }^{21}$.

L'Association of Headmistresses met en place un réseau international de directrices. Celles qui occupent un poste outre-mer sont admises au sein de l'association comme correspondantes, et non comme membres à part entière, bien qu'elles aient la nationalité britannique et même, dans de nombreux cas, une éducation universitaire anglaise, comme en témoignent le statut de membre de plein droit accordé à Miss Mary Douglas, directrice de Godolphin, et celui de correspondante octroyé à Miss Edwards et à Miss Ethel Jones au moment où elles rejoignent leurs postes de directrices en Afrique du Sud. En tant que correspondantes, les directrices des colonies reçoivent les publications de l'Association, mais ne peuvent prendre part aux votes lors des débats et des élections à l'assemblée annuelle. Durant leurs congés en Angleterre, elles ont le droit de participer en tant que membres à part entière à titre temporaire et sont autorisées à voter lors de la rencontre annuelle, mais perdent ce droit de vote lié à l'adhésion de plein droit lors de leur retour dans les colonies. Le droit de vote, le statut de membre à part entière, et donc l'autorité professionnelle, ont une dimension spatiale et sont situés géographiquement «au sein de la mère patrie » en Angleterre. Les catégories de statut existant au sein de l'Association reflètent la diversité des positions que sont susceptibles d'occuper ses membres vis-à-vis de la société métropolitaine ${ }^{22}$.

10 En tant qu'organisation professionnelle, l'Association s'attache à améliorer les conditions de travail, les salaires et l'avenir de ses membres ${ }^{23}$. Elle s'engage activement en 1912-1913 en prospectant auprès de ses correspondantes d'outre-mer pour obtenir des renseignements sur l'existence de postes disponibles à l'étranger pour des enseignantes de métropole et en sollicitant des informations sur les conditions de travail dans les établissements scolaires secondaires pour filles, sur les salaires, sur le coût de la vie et sur les qualifications universitaires et professionnelles requises ${ }^{24}$. Les difficultés rencontrées par les enseignantes pour trouver du travail lors de leur retour en Angleterre, qui les poussent de prime abord à se montrer méfiantes à l'égard de l'émigration, constituent une question fondamentale pour les directrices dans les 
colonies. L'Association y répond en encourageant les directrices de métropole à employer des enseignantes ayant forgé leur expérience outre-mer. Cette recommandation favorise le développement d'une circulation à double-sens d'enseignantes et de directrices entre les institutions scolaires féminines anglaises et leurs homologues à l'étranger, dont rendent compte les profils de carrière de certaines de ces femmes, et dont les mouvements de personnel entre Godolphin et les établissements sud-africains de St Michael's School et de la Diocesan School constituent un exemple.

11 L'Association of Headmistresses adhère à une conception du professionnalisme qui met l'accent sur le service combiné au savoir-faire ${ }^{25}$. Pour un grand nombre de jeunes femmes actives professionnellement, l'engagement religieux est à la fois source de discipline de soi et d'idéaux élevés ${ }^{26}$. De nombreuses jeunes enseignantes éprouvent le sentiment fort d'avoir une mission, d'être des pionnières et d'ouvrir à d'autres de nouvelles possibilités. Le développement de communautés formelles et informelles, qui offrent un soutien, un compagnonnage, une respectabilité et des contacts importants, joue souvent un rôle central pour permettre aux femmes de mettre en œuvre leurs idéaux professionnels. Nombreuses sont les jeunes femmes actives professionnellement qui forment au cours de leurs carrières d'enseignantes des relations amicales destinées à durer toute leur vie. On voit également d'anciennes élèves partir à la sortie du pensionnat pour suivre une enseignante admirée et travailler avec elle, cette « relation inégale mûrissant en un rapport de soutien et d'admiration mutuels ${ }^{27}$. Les activités et l'esprit de l'institution Godolphin de Salisbury, ainsi que ses liens avec St. Michael's School de Bloemfontein et la Diocesan School de Grahamstown, illustrent le fonctionnement des réseaux professionnels au niveau des établissements scolaires, et la manière dont la mission - à la fois religieuse et impériale - se nourrit de notions de service et de devoir qu'elle contribue dans le même temps à alimenter, notions qui constituent des aspects importants des identités professionnelles des enseignantes et des idéaux auxquels on souhaite voir adhérer les jeunes filles appartenant aux upper middle classes.

\section{Godolphin School à Salisbury : la mission et l'Empire}

Godolphin School a été fondée et dotée financièrement en 1707 par Elizabeth Godolphin en tant que pensionnat destiné à de jeunes orphelines de bonnes familles. Le système de gestion a été révisé en 1886 afin d'offrir une éducation secondaire aux jeunes filles des middle classes ${ }^{28}$. En 1890, Mary Alice Douglas, une ancienne enseignante de la Alice Ottley School de Worcester entre en fonction comme directrice. Née en 1860, elle est fille d'un chanoine de l'Église anglicane et petite-fille d'un évêque de cette même confession. Trois autres sœurs Douglas, Edith, Janet et Lucy, l'assistent à Godolphin. Janet se tourne ensuite vers le travail social au settlement de Talbot House à Camberwell (communauté de travailleurs sociaux au sein de quartiers pauvres), tandis que Lucy fonde la Society of Women, qui offre une "aide spirituelle" dans les hôpitaux londoniens. L'un des frères de Mary, Arthur Jeffreys Douglas, est membre de la Universities Mission (Mission des Universités) en Afrique centrale, tandis que Gerald, un autre de ses frères, missionnaire lui aussi, devient évêque du Nyasaland ${ }^{29}$. 
13 Mary Douglas adhère à l'Association of Headmistresses en 1892 et s'investit de manière importante dans ses activités. Elle est membre du comité exécutif de l'association à partir de 1902, accueille sa Rencontre annuelle à Godolphin School en 1910, et occupe les fonctions de présidente de 1911 à 1913. Elle co-dirige un ouvrage collectif publié en 1911, intitulé Public Schools for Girls, qui rassemble des essais rédigés par des directrices. Elle prend part à des activités professionnelles très diverses, et s'investit en particulier au sein du Teachers Registration Council (1912-20) (Conseil d'enregistrement des enseignants), du Consultative Committee of the Board of Education (à partir de 1912) (Comité consultatif de la commission pour l'éducation), du Salisbury Education Committee et $\mathrm{du}$ conseil d'administration de la Bishop Wordsworth School de Salisbury. Elle appartient à la commission coloniale de l'Association of Headmistresses; elle est en outre membre du comité exécutif de la Colonial Intelligence League ${ }^{30}$.

Les biographies des administrateurs de Godolphin School révèlent des implications similaires à celle de la famille Douglas dans les activités missionnaires et impériales. Composés en proportions variables de pédagogues, d'hommes d'affaires, de parents, d'anciens élèves et de membres de la communauté, hommes et femmes, les conseils d'administration des écoles anglaises sont des instances devant lesquelles le directeur doit répondre de sa gestion de l'établissement. Les administrateurs de Godolphin ont avec l'Empire des liens forts, similaires à ceux qu'entretient avec lui la directrice Mary Douglas, dont ils surveillent la gestion de l'école. L'évêque Webb, doyen de Salisbury, est ainsi ancien évêque de Bloemfontein ${ }^{31}$, tandis qu'à partir de 1913, la fonction de président du conseil d'administration est occupée par le maréchal lord Methuen, commandant en Afrique du Sud pendant la Guerre des Boers puis commandant en chef à partir de $1908^{32}$. Sarah Maria Fawcett a, quant à elle, des liens familiaux avec l'Afrique du Sud par le biais de sa belle-sœur, la militante suffragiste Millicent Fawcett, qui dirige la commission de femmes envoyée par le Gouvernement britannique en Afrique du Sud pour contrôler les conditions de détention dans les camps de concentration de la Guerre des Boers. Millicent emmène sa fille Philippa, qui est aussi la nièce de Sarah. Toutes deux retournent en Afrique du Sud en 1903, Millicent en tant que conférencière de la Victoria League et Philippa pour mettre en place «un appareil éducatif permanent» pour l'éducation élémentaire ${ }^{33}$. Autre membre du conseil, lady Hulse voyage un peu partout à travers l'Empire; elle est l'amie de lady Milner, épouse du Gouverneur général d'Afrique du Sud ${ }^{34}$. Quant à lady Everett, sa nièce May Wyld, ancienne élève de Godolphin, est la Principale de Hahbubia Girls' School, en Inde, à Hyderabad $^{35}$. Étant donnée la responsabilité des administrateurs en matière d'organisation de la stratégie générale de l'école, de tels liens avec l'Empire sont importants.

Godolphin School est pénétrée d'un esprit profondément religieux. Mary Douglas complète l'œuvre de ses frères missionnaires. Elle accorde la première place à la formation spirituelle et à celle de la personnalité, et, pendant de nombreuses années, se charge de l'enseignement religieux dans toutes les classes de l'établissement. Elle rédige le livre de prières de Godolphin, se passionne pour le chant sacré, organise un festival de chant à l'école et raconte de manière très vivante des histoires de l'Ancien Testament à Hall Divinity. Chaque mardi matin, on prie pour les missions étrangères, tandis que toute une série de jeunes filles noires d'Afrique de l'Ouest sont "adoptées " et rebaptisées. Des quêtes en faveur des missions ont lieu, de même que des visites régulières de missionnaires. Un certain nombre d'évêques de l'étranger, tant blancs 
qu'indigènes, visitent l'établissement, tandis que des femmes viennent parler de leur travail de missionnaires en Angleterre et dans les colonies ${ }^{36}$.

Les jeunes filles sont directement appelées à s'investir dans le travail de mission. Secrétaire pour les écoles de la Society for the Propagation of the Gospel (Société pour la propagation de l'Évangile), l'administratrice Miss Moberley harangue les élèves de Godolphin sur le thème des missions étrangères. "Encore et encore, elle nous inculquait le fait que l'on avait partout besoin de gens prêts à travailler, de femmes en particulier", se souvient l'une d'elles ${ }^{37}$. Les missionnaires en visite à Godolphin enfoncent le clou. Le Révérend R. G. Douglas « était particulièrement désireux de nous pousser à nous intéresser davantage au travail de Mission » : « Il comprit que personne à Godolphin n'était pour le moment allé travailler en terre de mission. Il espérait que parmi nos distinctions nous compterions un jour le grand honneur d'avoir parmi nous une pionnière ouvrant une voie qui ferait des émules $»^{38}$.

Les élèves et les anciennes de Godolphin participent à la United Girls' School Mission (Mission de l'union des écoles de filles), dont Mary Douglas préside le comité exécutif. Cette mission est fondée à l'initiative d'un certain nombre d'écoles de filles en 1897 à Camberwell, un quartier surpeuplé de Londres. Elle reçoit le soutien financier des écoles de filles et, en particulier, de leurs associations d'anciennes, et fonctionne sur le mode des settlements. L'œuvre missionnaire est effectuée par le clergé, aidé de laïcs, hommes et femmes. Un centre de formation destiné aux anciennes élèves désireuses de se préparer au travail social est associé au settlement de Camberwell. Cette mission constitue l'un des moyens par lesquels les élèves et les anciennes de Godolphin peuvent apprendre "quelque chose sur les problèmes sociaux et le service social dans les centres urbains surpeuplés ». Des quêtes hebdomadaires ont lieu à l'école en faveur de la mission, tandis que Mary Douglas note que la prière quotidienne pour la mission " concourait à rendre [celle-ci], consciemment ou inconsciemment, partie intégrante de l'air de Godolphin que nous respirions " ${ }^{39}$. Deux anciennes de Godolphin se succèdent au poste de secrétaire chargée de l'organisation de la mission et reviennent à l'école parler du travail qui y est effectué.

D'autres se rendent à Godolphin pour présenter les perspectives qui existent au sein de l'Empire. M. Lloyd, directeur du Fellowship of the Maple Leaf (Corporation de la feuille d'érable), en appelle aux enseignantes pour "garder le Canada britannique et chrétien ». DameMerial Talbot présente une conférence sur la Victoria League, tandis qu'on compte également parmi les visiteuses lady Edward Cecil (plus tard vicomtesse Milner). Les élèves et les maîtresses de Godolphin s'enorgueillissent d'avoir une place à la tribune lors de la première célébration de l'Empire Day à Salisbury en 1909, devant les enfants de la ville rassemblés après avoir fêté ce jour dans leurs écoles. Empire Day, célébré le jour de l'anniversaire de la reine Victoria, avait été érigé en fête nationale au Canada en 1901 ; des propositions visant à étendre cette fête à l'Angleterre avaient été inscrites à l'ordre du jour de la Colonial Conference (Conférence coloniale) de 1902. Ce jour-là, des groupements impériaux comme la League of Empire et la Victoria League, de même que des organisations impérialistes de jeunes, associent leurs actions ${ }^{40}$. Lors de la première célébration à Salisbury, c'est une administratrice de Godolphin, lady Hulse, qui déploie le drapeau sur la place du Marché de la ville. En 1914, les prières précédant la marche pour se rendre aux cérémonies sont l'occasion pour Mary Douglas de rappeler aux élèves «les quatre mots d'ordre de notre Empire - la responsabilité, qu'imposent les privilèges, la compassion, le sacrifice de soi et le devoir $»^{41}$. 
19 La religion et l'Empire fusionnent dans les vertus de la «féminité impériale» transmises aux filles. Lors de la remise annuelle de diplômes en 1902, le doyen de Salisbury et administrateur de Godolphin, s'adresse aux élèves en les qualifiant de «filles de l'Empire »: «Lord Rosebery dit que le sentiment impérial devrait conférer non pas de l'arrogance, mais le sens des responsabilités. Ici, dans cette école à l'ombre de la Cathédrale et à proximité de Stonehenge... qu'allez-vous apporter à l'Empire ? Pas la mode, pas les cancans, pas l'argot de la bonne société, pas les horribles types de femmes qu'on voit dans les romans de mœurs, mais le genre de filles décrites par Miss Douglas, pénétrées de sentiments, mais non de sensiblerie - car l'Empire est soudé par le sentiment, la loyauté et l'humanité, qui sont les qualités qui ont conduit les Canadiens et d'autres fils de l'Empire à nous venir en aide dans la guerre actuelle ${ }^{42}$. Dans le discours qu'il prononce l'après-midi du même jour, il met l'accent sur des notions religieuses de service, et appelle les jeunes filles à être prêtes à servir et à assumer un sacerdoce en tant que "filles du grand Roi » ${ }^{43}$. Une telle rhétorique fait partie des idéaux de service, de sacrifice et de persévérance que Martha Vicinus présente comme associés à des notions de classe et de supériorité sociale ${ }^{44}$.

20 À la date de 1926, cinquante-trois anciennes élèves vivent en Afrique, quarante-quatre en Inde, vingt-deux au Canada, treize en Australie, huit en France, sept en Chine, six aux États-Unis, cinq en Nouvelle-Zélande, de même qu'en Égypte et au Soudan, trois en Belgique, en Allemagne et en Suisse, deux au Japon et en Argentine, et une enfin respectivement au Cap Vert, en Hollande, en Italie, en Irak, au Mexique, en Palestine et en Sibérie ${ }^{45}$. Les enseignantes sont, elles aussi, parties à l'étranger, certaines pour occuper des postes à la tête d'établissements secondaires pour filles. Le grand nombre d'anciennes qui s'installent en Afrique du Sud s'explique en partie par les liens des administrateurs et de la famille Douglas avec ce pays, ainsi que par la position favorable à la guerre contre les Boers de ceux qui sont associés avec l'école. L'influence des opinions politiques des administrateurs sur l'esprit de l'établissement ne doit pas être sous-estimée. Comme le montre l'exemple d'Edith Creak, qui dirige la King Edward VI School à Birmingham, l'existence de divergence d'opinions politiques entre les administrateurs et la directrice peut amener celle-ci à se retrouver confrontée à de réelles difficultés. À la différence de ce qui se passe à Godolphin, où administrateurs et directrice se rejoignent sur les questions touchant l'Empire, Edith Creak se retrouve en désaccord avec le conseil d'administration de son établissement, qui ne partage pas sa position lorsqu'elle se déclare ouvertement en faveur de la guerre des Boers. Elle fait alors l'objet d'une réprimande officielle et est sommée de retirer sa déclaration publique, tandis qu'il lui est interdit d'aborder le sujet de la guerre aussi bien à l'intérieur qu'à l'extérieur de son établissement de Birmingham ${ }^{46}$.

21 Quand 10000 hommes sont envoyés au Natal en 1899, le magazine de Godolphin publie un témoignage sur l'embarquement des troupes à bord de navires à proximité de Southampton ${ }^{47}$. Les notions de patriotisme et de sacrifice de soi caractérisent le discours que prononce Mary Douglas devant son école sur la guerre au Transvaal, où sont partis combattre les frères de plusieurs élèves: "Après avoir esquissé l'histoire passée du Transvaal et évoqué le déroulement de la guerre, elle parla des bienfaits que l'on pourrait retirer d'un tel conflit, dus aux sentiments nobles de patriotisme et de sacrifice de soi qu'il suscite. Elle termina en disant [...] que nous devrions saisir avec joie toute occasion de faire le petit peu qui est à notre portée pour soulager la douleur et la souffrance que doit inévitablement causer la guerre. À la fin de la séance, on 
chanta le God Save the Queen... Depuis cette réunion, nous avons dit des prières chaque jour pour ceux qui combattent et souffrent, et une quête est organisée chaque semaine en faveur des malades et des blessés $»^{48}$.

En 1900, la levée du siège de Ladysmith est célébrée, et le journal de Godolphin rend compte du retour de navires et de soldats. En 1902, Mary Douglas emmène toute l'école assister à la lecture par le maire de la proclamation de la paix. L'évêque Ridgeway parle des camps de concentration de la Guerre des Boers devant les élèves et le personnel, tandis que Millicent Fawcett visite l'établissement pour donner une conférence sur ses expériences sud-africaines ${ }^{49}$. Lors de la Rencontre annuelle de 1913, Mary Douglas évoque la nomination de lord Methuen à la tête du conseil d'administration de l'école comme « quelque chose qui fait vibrer l'âme et enflamme l'imagination, et nous donne le sentiment que nous sommes par là liées avec quelqu'un qui a combattu en territoire Ashanti, sur la Côte de l'Or, en Égypte, en Afrique du Sud, quelqu'un qui, dans un sens qui n'a rien de simplement métaphorique, a supporté le fardeau et la violence de l'époque, et qui a remporté partout les récompenses dues à la valeur ${ }^{50}$.

Des liens précoces tant avec la Diocesan School de Grahamstown qu'avec St Michael's School de Bloemfontein se mettent en place dès avant le Pan-Anglican Congress de 1908, mais se développent surtout de façon importante au cours des années qui suivent ce congrès, auquel assistent des membres du personnel de Godolphin ainsi que des élèves et des anciennes. Mary Douglas intervient dans la section du congrès consacré à « La préparation des jeunes à servir les autres au sein de l'Église ». On l'applaudit pour sa conviction que «la vie scolaire recèle les plus magnifiques possibilités d'offrir un enseignement qui serait fécond dans le cadre d'une vie consacrée au service... [et] que le plus gros du travail se fait au cours de la routine ordinaire qui allie occupation, travail et récréation $»^{51}$. Le Pan-Anglican Congress organise également une rencontre pour les jeunes des deux sexes au Albert Hall de Londres. Ils y sont harangués par des évêques de métropole et des colonies, qui leur parlent de «la vocation à servir notre pays", de "la vocation à servir notre Empire" et de la vocation à devenir missionnaires «au sein de la mère patrie». La notion d'un service qui serait "immédiat, dévot et personnel» constitue l'un des thèmes de cette rencontre organisée à l'intention des jeunes ${ }^{52}$. Le congrès influe de fait sur la décision de plusieurs membres de Godolphin de s'engager dans le travail de mission. Miss Ruth Wordsworth, Susie Steedman, Miss Jones et Miss Edith Douglas se rendent ainsi toutes à Wandsworth pour se former à ce type d'action.

Le thème de "l'extension de la famille de Dieu » à l'ensemble de la planète, qui soustend la rencontre organisée pour les jeunes des deux sexes au Albert Hall dans le cadre du congrès, a une longue tradition à Godolphin. Dans son discours aux élèves et aux anciennes lors de la fête de Commémoration (Commem.) de 1903, Arthur Douglas relie l'amour de l'école et la loyauté à son égard à une vision idéale de l'école comme famille chrétienne: "Vous n'êtes pas seulement des filles de Godolphin: vous êtes des filles chrétiennes. Vous portez non seulement l'insigne de l'École à vos chapeaux, mais aussi l'insigne des Chrétiens à vos fronts. Vous appartenez à Godolphin School, et à l'École du Christ. Cette école est un terrain d'apprentissage en matière de formation du caractère, et je pense que beaucoup d'entre vous considèrent leur École davantage comme un second foyer, comme si vous formiez toutes une seule et même famille. L'École chrétienne est en fait une famille. Nous sommes tous les enfants de notre Père qui est aux Cieux : nous sommes membres de la famille de Dieu. Nous avons reçu des dons de 
notre Père céleste et nous avons des devoirs envers Lui. Au sein de la famille du Père céleste, comme au sein de la famille de Godolphin School, maintenez des liens vitaux et des contacts par la prière et la Sainte Communion $»^{53}$.

Le fait de rester en contact et de construire un réseau d'anciennes de Godolphin incluant le personnel et les élèves est sous-tendu par des impératifs spirituels, et se traduit en "action" à travers des rituels tels que la Commem., qui joue un rôle fondamental au sein de la " communauté imaginée » internationale qu'est Godolphin ${ }^{54}$. La Commem. réunit les administrateurs, le personnel, les élèves et les anciennes. Elle est présente à l'esprit de celles qui se trouvent dans des pays éloignés, qui envoient des télégrammes pour marquer l'événement ${ }^{55}$. Lors de la Commem., les liens de loyauté envers la communauté de Godolphin et ses idéaux sont réaffirmés, l'école est évoquée comme une véritable famille, tandis que garder le contact avec elle est présenté comme un devoir chrétien : "Maintenant l'École vous pose la question : 'qu'avez-vous fait pour moi ?' Faites-en plus à l'avenir : faites rendre vos dons avec intérêts, pour payer l'École en retour; continuez de suivre toutes les traditions élevées et les idéaux que vous y avez appris, où que vous soyez, afin de ne pas marquer d'une tache l'écusson de l'École ; maintenez-le pur et propre toute votre vie. Restez en contact avec vos vieilles amies; faites preuve de sympathie à l'égard des autres anciennes. Restez au courant des intérêts de l'École, maintenez des liens vitaux et des contacts, sous des formes autres que simplement assister à la Commem. ; continuez de répondre à ses appels. Continuez de prier pour l'École. Alors vous comprendrez que recevoir des dons implique des responsabilités. Vous vous en retournerez en réalisant plus que jamais auparavant tous les bienfaits que vous a apportés l'École, et vous consacrerez vos plus grands efforts à être loyales et fidèles à ses intérêts $"^{56}$.

L'appel au devoir en tant que membre de la famille de Godolphin est formulé en un langage à la fois religieux et militariste. Il fait fortement appel à l'émotion et est qualifié par les élèves et les anciennes de «frisson de la Commem. ». "Précisément à des moments comme celui-ci, à la Commémoration, je crois qu'on sent vraiment qu'on est toutes membres d'une même et grande École, et que celle-ci est tout simplement la plus splendide École du monde. Et puis il y a ce sentiment que nous éprouvons, parfois, on l'appelle 'le frisson de la Commem.', ne nous pousse-t-il pas à vouloir faire quelque chose ? ... Parfois, je pense que nous sommes arrivés au moment le plus critique de l'histoire du monde - à coup sûr de l'histoire du grand Empire auquel nous appartenons ; partout les forces du bien et du mal s'agitent de toutes parts. Pensez à ce que ça signifierait si un grand bloc solide comme Godolphin School combattait pour le bien tout autour de lui ; si nous pouvions seulement nous y mettre énergiquement et combattre d'une seule âme pour tout ce qui est vrai et pur, et beau, et de bon renom... Ça signifierait plus qu'on ne peut ne serait-ce qu'imaginer. La Commem. va s'achever, et le 'frisson' s'évanouira, comme la splendeur du ciel au couchant du soleil ; mais nous, ne nous souviendrons-nous pas de ce que nous avons vu, et ne nous lancerons-nous pas dans la bataille avec un grand courage et 'les visages des matins rayonnants' ? ${ }^{57}$

Avec l'expansion en Afrique du Sud de la communauté d'anciennes élèves et de membres du personnel de Godolphin, les idéaux de l'école sont mis en œuvre dans ce pays. Les jeunes filles se tiennent sur le quai pour accueillir les nouvelles arrivantes, tandis que les enseignantes établissent des rapports de sociabilité avec leurs anciennes élèves. Alors qu'elle était encore en pleine mer en direction de l'Afrique du Sud - à 1000 miles du Cap -, Miss Jones reçoit ainsi un marconigramme avec les mots: 
«Bienvenue de la part des Godolphiniennes ». Elle passe quelques temps au Cap avec Edith Soloman, qui « va très bien et prend du bon temps en jouant au tennis et en allant au bal, à côté de toutes sortes de travaux qu'elle effectue dans la maison». Edith l'emmène à Camps Bay et à Rondebosch visiter Groote Schuur, la maison de Cecil Rhodes. Miss Jones voit Joan Evans, qui est une " fille d'intérieur on ne peut plus utile", enragée de golf et "très avide de toutes sortes de nouvelles de Godolphin ». Thyra Pierce, une ancienne élève devenue une maîtresse de musique très occupée, fait une apparition au Cap pour faire ses adieux sur le bateau : "Ces trois-là sont aussi loyales qu'on peut l'être à leur vieille École $»^{58}$. En 1910, un télégramme de Commem. portant les noms de seize anciennes élèves et maîtresses de Godolphin est envoyé de Bloemfontein. Deux ans plus tard, sept anciennes Godolphiniennes, enseignantes et élèves, se retrouvent dans la chambre de Miss Edwards à St Michael's School de Bloemfontein pour célébrer la Commem. Le partage d'un rituel les lie à la "communauté imaginée» des Godolphiniennes d'Angleterre, leurs boutonnières rouges et blanches étant "nouées du ruban rouge et blanc adéquat ». Elles évoquent «en grands détails» leurs années d'école et la Commémoration. Petit à petit, la conversation s'étend à d'autres sujets, « mais le frisson de la Commem. était là tout du long $»^{59}$.

Des lettres vont et viennent entre anciennes et membres du personnel de Godolphin, créant une circulation à double-sens d'informations et d'idées qui traverse les frontières métropolitaines. Le journal de l'école publie régulièrement des lettres d'Afrique du Sud. Des visites aux Godolphiniennes sud-africaines par le personnel, les anciennes élèves et leurs parents facilitent le passage d'informations en Angleterre. En 1907, le journal rapporte des nouvelles de Miss Edwards provenant de M. Short, « le frère de notre Miss Short », et de Miss Malden, «la cousine de notre Miss Malden », tous deux juste de retour de Bloemfontein. «Ils nous disent que Miss Edwards se porte merveilleusement bien et qu'elle fait des miracles dans son école. Une nouvelle maîtresse d'école maternelle, qui a été formée par notre ancienne, Lucy Bury, à Winchester, est partie la rejoindre, de même qu'une professeur de gymnastique suédoise, tandis que notre Miss Egerton va enseigner le français et l'allemand $»^{60}$. Quittant Godolphin où elle enseignait, Grace Bagnall fait étape en Afrique du Sud pour une visite, sur la route de son nouveau poste à la Diocesan School d'Auckland, en Nouvelle-Zélande. Miss Ralph fait de même lors de son retour en Angleterre, après avoir occupé le poste de directrice de St Margaret's en Colombie britannique ${ }^{61}$. Grace Bagnall écrit du Cap : «L'endroit semble vraiment plein de gens de Godolphin ${ }^{62}$.

La figure du Home, de la Patrie, du chez soi, est très présente dans de nombreuses lettres d'Afrique du Sud ${ }^{63}$. En 1908, Miss Jones écrit ainsi : « Les gens sont extrêmement intéressants [...]. J'ai été frappée tout du long par le fait qu'ils se sentent si proches de chez eux. Tous viennent d'endroits qu'on connaît. Tous ont prévu de rentrer chez eux un jour. Tous écrivent à la maison chaque semaine, et en reçoivent des nouvelles à chaque arrivée de courrier. Tout vient de chez nous $»^{64}$.

L'école en tant que "chez soi » est très présente dans des lettres qui construisent de manière performative le Home comme antithèse de l'Altérité que représente l'Empire. À son arrivée à la Diocesan School de Grahamstown, Miss Jones écrit ainsi : «Et pendant tout ce temps Miss Edith accueille mes Anciennes bien-aimées à Nelson et je n'y suis pas. Moi, je suis ici en train de m'y rendre dans la voiture de M. le Juge Hutton [...]. On m'offre un bain chaud et une tasse de thé quand j'arrive à l'École, juste au moment où 
toutes les Anciennes se rendent dans la Classe de Miss Douglas [...]. Samedi matin j'assiste à l'Office et vous êtes toutes à St Martin's ; je brûle d'y être [...]. Mon cœur est à Salisbury... $»^{65}$.

31 Beaucoup des discours sud-africains se fondent sur des idéaux d'identité et de supériorité anglaises. Le journal décrit la campagne d'Afrique du Sud, ses bâtiments, sa vie scolaire et ses élèves indigènes en des termes familiers et faisant appel au langage de la supériorité culturelle : «Les filles de la [classe] VI travaillent pour les Matric. du Cap (examens), qui ne sont pas aussi difficiles que le Higher Certificate (diplôme supérieur) mais l'anglais, les mathématiques, le latin et une langue moderne sont tous obligatoires et doivent être passés en même temps. La V prépare le 'School Higher' (examen supérieur de l'École), un examen qui ne paraît pas vraiment mortel à des yeux anglais, mais les enfants ici n'ont pas la chance que vous avez à la maison, heureuses comme vous êtes [...] il n'y a pas d'old Sarum, ou de Clarendon, ou d'Abbaye de Westminster, ou de Tour de Londres pour leur apprendre l'histoire, et que peuventelles savoir de la géographie dans ce pays, où la rivière la plus proche est à des miles, alors que la plupart d'entre elles n'ont jamais eu l'occasion de voir la mer. Oh, vous les enfants si chanceuses de chez nous, rendez grâce chaque jour de votre vie pour toutes les belles choses que Dieu a faites ou que l'homme a inventées, qui vous entourent de tous côtés... $»^{66}$.

Dans les lettres envoyées à Godolphin se perçoit fréquemment l'aspect personnel des relations que nouent les femmes à travers leur travail dans l'Empire ${ }^{67}$. Le fait d'être dans les colonies offre cependant de nouvelles possibilités en matière de rapports entre les enseignantes et les élèves restées en métropole. Ceux-ci reposent sur une « règle de la différence coloniale" complexe, car recouvrant des formes différenciées d'appartenance aux populations blanches (anglaises, hollandaises et juives) «fondées sur le pouvoir que la métropole exerce sur 'ses' peuples-sujets $»^{68}$. Sur un ton familial rappelant celui d'une sœur aînée, Miss Edwards, la directrice de St Michael's, fait des filles de Godolphin ses confidentes en leur décrivant les élèves de son établissement. Adopter la position de "grande sœur " à l'égard des filles de Godolphin permet à Miss Edwards de maintenir une distance entre ces dernières et elle-même, tout en les plaçant dans une position de supériorité par rapport aux élèves de St Michael's : « Il y a Minnie Galpin, la prefect (élève des grandes classes chargée de la discipline), pas très intelligente, mais une fille très gentille, qui ressemble assez physiquement à Mary Liepmann; Gladys Ehrlich, une petite Juive au visage éveillé et aux yeux bruns, qui travaille sans relâche comme une Troyenne; Elfie Baumann (pron. Bowmann), sa cousine mais avec des yeux gris et des cheveux bruns raides, séparés au milieu par une raie et pendant en mèches folles de chaque côté... Je peux vous dire qu'Elfie est une ensorcelante et incorrigible petite coquine, qui danse un peu partout dans la plus courte et flottante des jupes, et produit généralement une impression d'agitation des bras et des jambes, elle est observatrice, se rappelle des choses et réfléchit, mais elle fait le désespoir de sa maîtresse, parce qu'elle ne s'arrête pas à des détails tels que celui de rendre un travail que, parfois, il lui arrive d'avoir fait. J'ai bien peur qu'elle ne vous plairait que trop $»^{69}$.

33 Elle fait connaître aux élèves les « aspects compliqués » de la " question des races ", ses lettres faisant état de nombreux niveaux de différenciation culturelle ${ }^{70}$. « À Durban et au Natal, la question des races est compliquée par la présence d'Indiens. On les a fait venir à l'origine pour travailler dans les plantations de cannes à sucre, et maintenant ils 
sont propriétaires de la plupart des jardins maraîchers, et travaillent sur les routes. Les Musulmans ont des magasins de tissus. Ils forment un peuple très pittoresque, gracieux et beau. Les couleurs de leurs tissus sont on ne peut plus belles, et je n'ai rien vu de plus pittoresque que leur marché dans une petite cour baignée de soleil contiguë à leur mosquée - ni rien de plus impressionnant qu'un service religieux dans cette même mosquée. $»^{71}$

Lors d'un séjour qu'elle effectue en Angleterre en 1909, le discours qu'elle tient à Godolphin reconnaît à la «métropole » une position de supériorité. À la manière des autres commentateurs, hommes et femmes, elle présente l'Afrique du Sud comme un pays vide, dépourvu de culture, dont l'« histoire » est écrite selon des normes anglaises et vue à travers un regard occidental et sexué, tout ceci contribuant simultanément à situer "les races de couleur" au sein d'une "grammaire» de la différence ${ }^{72}$. "Dans l'énorme pays qu'est l'Afrique du Sud, il n'y a guère d'histoire sur laquelle on puisse faire un retour, elle est toute entière en train de se faire. Il faut faire face à d'énormes problèmes: comment vivre et mener une guerre victorieuse contre la fièvre, les criquets, la sécheresse et les maladies épidémiques; comment trouver l'argent nécessaire au développement du pays; comment travailler à la paix entre les Néerlandais et les Anglais; comment résoudre le problème prodigieusement plus important des races de couleur - indigènes, Malais, Hindous. Dans le même temps, les enfants grandissent et il faut les éduquer. Ils n'ont aucune des choses, riches de beauté et de sens, qui vous aident en Angleterre - les images, les bâtiments, la musique et le souvenir des vies des grands hommes $»^{73}$.

\section{III. École, Association et « règle de la différence »74}

Les trois établissements scolaires et l'Association of Headmistresses sont impliqués dans la construction de hiérarchies portant sur le genre, la race, le groupe ethnique et l'appartenance de classe qui permettent de fixer les frontières entre sujet et citoyen, ou encore entre " mère patrie ", colonie, nation et Empire, à travers une "règle complexe de la différence coloniale ». Cette règle est l'une de celles par lesquelles l'Angleterre a "imaginé » et administré son Empire. Les sujets blancs d'Angleterre ont un statut différent des colons blancs de pays comme la Nouvelle-Zélande et l'Australie, dont le statut évolue vers celui de dominion à la fin du siècle. Ces mêmes colons se différencient des sujets de couleur de pays comme l'Inde ou les Antilles, où, dans les dépendances britanniques, la maturité politique des populations noires majoritaires est considérée comme appartenant au futur, la Grande-Bretagne assumant la responsabilité du pouvoir au bénéfice de ceux qu'elle voit comme "inaptes » à se gouverner eux-mêmes. Les hiérarchies de genre, parallèlement à celles de classe, de race et de groupe ethnique, font partie de cette «règle complexe de la différence coloniale ", qui met en rapport la nation et l'Empire à travers les identités culturelles associées aux différentes formes d'appartenance nationale. De nombreux mécanismes de différenciation de statut des sujets sont informels plutôt que formels. Les multiples niveaux discursifs mis en œuvre par les femmes dans le contexte de l'Empire constituent l'un des aspects informels de cette « règle de la différence ».

Les appels à servir au sein du réseau chrétien de la communauté de Godolphin School diffusent un «type idéal» de jeune fille anglaise et d'enseignante du secondaire. Les discours sur la loyauté et le service envers l'école s'inspirent de notions emblématiques 
du Home et de la famille qui s'expriment à travers les idées de service religieux et de responsabilité vis-à-vis de l'Empire, sous des formes liées à une appartenance de classe et donc sources d'exclusion. À travers des discours et des rituels inventés (notamment la Commémoration), la «communauté imaginée » de Godolphin en Angleterre et en Afrique $\mathrm{du}$ Sud se construit par le biais du partage de signes et de notions d'appartenance et d'altérité mises en œuvre de manière performative, qui se nourrissent de conceptions de la nation qu'elles alimentent dans le même temps ${ }^{75}$. Dans la circulation à double-sens d'informations entre Godolphin et l'Afrique du Sud, le Home se construit comme une antithèse de l'Altérité que représente l'Empire, vu à travers le prisme culturel d'un regard anglais. Les notions de «Chez nous » et d'« Ailleurs » ne se construisent cependant pas dans des espaces métropolitains et coloniaux hermétiquement clos. Alors que les enseignantes communiquent à travers la distance, des visions divergentes de la féminité apparaissent ${ }^{76}$, ce dont les descriptions des élèves sud-africaines envoyées à Godolphin donnent un bon exemple : rédigées sous une forme compréhensible par les filles de Godolphin en Angleterre, elles n'en sont pas moins construites autour d'une idée de différence culturelle. Cet échange d'informations illustre la manière dont l'Empire peut se concevoir comme un "cadre structurant des échanges politiques, économiques et culturels entre la métropole et les colonies au sein d'un réseau d'interactions", plutôt que comme un ensemble discontinu d'États-nations et de colonies ${ }^{77}$.

37 La circulation transnationale d'informations s'inscrit profondément dans les stratégies de professionnalisation de l'Association of Headmistresses, stratégies qui construisent des identités professionnelles et un savoir-faire à travers de "précieux échanges d'expérience " $^{78}$. Les trois directrices - Mary Douglas à Salisbury, Gertrude Edwards à Bloemfontein et Ethel Jones à Grahamstown - appartiennent toutes à la «famille» de Godolphin. Seule Mary Douglas, géographiquement en métropole, est pourtant membre de l'Association à part entière. Les débats sur les critères à remplir pour intégrer l'Association of Headmistresses, sur les termes de cette intégration, et sur l'octroi ou non du droit de vote et de participation à la gestion de l'association, à la fois se fondent sur, et renforcent, des frontières et des hiérarchies entre "sujet et citoyen, 'mère patrie', colonie, nation et Empire ", en « reconnaissant » des identités de colonisateur/colon blanc colonisé à travers le statut de correspondant étendu à des femmes comme Miss Edwards et Miss Jones, de nationalité anglaise mais installées en Afrique du Sud. Dans ses lettres aux filles de Godolphin, Miss Edwards nuance davantage encore les positions des sujets blancs en dépeignant la culture anglaise et les élèves de Godolphin comme supérieures à la culture sud-africaine et aux élèves anglaises, hollandaises et juives de St Michael's School. Elle établit également une distinction entre les identités culturelles anglaise, hollandaise et juive d'Afrique du Sud et les positions de sujets qui sont celles des « races de couleur - indigènes, Malais, Hindous ». Ces distinctions renforcent d'une façon informelle les différenciations qui sont au cœur de la "règle de la différence coloniale ", à travers laquelle les sujets blancs d'Angleterre, les colons blancs et les populations noires majoritaires sont imaginés et administrés par l'État britannique. Des hiérarchies liées au genre jouent un rôle central dans ce processus informel. La construction de l'école comme une famille et un chez soi, les visions de la féminité mises en avant et la position de grande sœur adoptée par Miss Edwards, avec ce qu'elle implique en termes de relations de pouvoir entre femmes, forment des axes centraux autour desquels est imaginée, entretenue et maintenue une "règle de la différence coloniale » que les 
enseignantes et les élèves mettent en œuvre dans les espaces culturels où se joue l'éducation des filles, espaces qui assurent une liaison entre les colonies et l'Empire ${ }^{79}$.

\section{NOTES}

1. M.A. Douglas, C.R. Ash : The Godolphin School, 1726-1926, London, Longmans Green and Co, 1928, p. 227 ; Godolphin Magazine, $\mathrm{n}^{\circ} 19,1901$, pp. 26-27 ; n³0, 1905, p. 24; $\mathrm{n}^{\circ} 31,1905$, p. 4 ; n 37, 1907, p. $9 ; n^{\circ} 38,1907$, p. $18 ; n^{\circ} 44,1909$, p. $31 ; n^{\circ} 45,1910$, p. $15 ; n^{\circ} 50,1911$, p. $35 ; n^{\circ} 52,1912$, p. 7 ; n 53, 1912, p. 19.

2. Godolphin Magazine, $\mathrm{n}^{\circ} 12,1899$, p. 24 ; n 19, 1901, p. 24 ; $\mathrm{n}^{\circ}$ 53, 1913, p. 4 ; n 57, 1914, p. 4.

3. A. MacKinnon: Love and Freedom: Professional Women and the Reshaping of Personal Life, Cambridge, Cambridge University Press, 1997 ; M. Theobald : Knowing Women : Origins of Women's Education in Nineteenth-Century Australia, Cambridge, Cambridge University Press, 1996, p. 48 et p. 105 ; K. Morris Matthews : For and About Women: Women's Studies in New Zealand Universities, 1973-1990, Thèse de Ph.D. non publiée, University of Waikato, 1993, p. 71 ; W. Robinson : « Women and Teacher Training: women and pupil-teacher centres, 1880-1914 ", in Women, Educational Policy-Making and Administration in England: Authoritative Women since 1800, sous la direction de J. Goodman, S. Harrop, London, Routledge, 2000, p. 106.

4. M. Theobald : Knowing Women..., op. cit., p. 48.

5. Ibid., p. 48 et p. 105.

6. Joyce Goodman: «A diaspora of highly educated women: women career teachers, space, networks and geographies of Empire", et Kay Morris Matthews: "Centre and periphery/ networks and spaces: 'imagining home' - women graduate teachers abroad, 1880-1920». Ces deux communications ont été présentées à la Joint British History of Education Society and Australian and New Zealand History of Education Society Conference, qui s'est tenue à Swansea en 2002.

7. M. Theobald : Knowing Women..., op. cit., p. 48.

8. http://www.sac.ecape.School.za/dsgweb/welcome.htm (téléchargé le 16/11/01). H. O'Connor : No Other School So Dear. A History of the Eunice Schools 1875-1970, Bloemfontein, imprimé à titre privé, s.d. (fin des années 1980), p. 9.

9. M. Price, N. Glenday : Reluctant Revolutionaries: A Century of Headmistresses 1874-1974, Bath, Pitman, 1974, p. 1 et p. 62.

10. AHM, comité exécutif, 11 mai 1897 ; les procès-verbaux du AHM ont été consultés au Warwick Modern Records Centre.

11. Ibid., 9 juillet 1904.

12. James G. C. Greenlee : «The ABC's of Imperial Unity », Canadian Journal of History, $\mathrm{n}^{\circ} 14,1979$, pp. 49-64.

13. http://library.canterbury.ac.nz/mb/archives/mb367organ.shtml (téléchargé le 19/01/2003). Voir également http://khozi2.nu.ac.za/ead/VictorP.html(téléchargé le 19/01/2003).

14. AHM, Annual Report, 1910, p. 50 et p. 64 ; J. Bush : Edwardian Ladies and Imperial Power, Leicester, Leicester University Press, 2000, p. 144.

15. Eugene Stock : History of the Church Missionary Society, London, CMS, 1916, vol. 4, pp. 549-551.

16. Pan Anglican Congress of 1908 : Report of the Women's Meetings, London, SPCK, 1908, pp. V-VI.

17. J. Bush : Edwardian Ladies..., op. cit.,p. 78. 
18. AHM, comité exécutif, 12 octobre 1907.

19. AHM, « Notes of Discussion at the At Home given by the President at the Clapham High School on June $30,1908 »$.

20. Calendrier de la Colonial Intelligence League (CIL), 4, CIL AR, 1910-11, pp. 7-14.

21. Ibid.; AHM, AR, 1911, rapport du comité exécutif, p. 42.

22. C. Hall : "Histories, Empires and the Post-Colonial Moment", in The Post-Colonial Question: Common Skies, Divided Horizons, sous la direction de I. Chambers, L. Curti, London, Routledge, 1996, p. 72 ; C. Hall : "The Rule of Difference: Gender, Class and Empire in the Making of the 1832 Reform Act ", in Gendered Nations : Nationalisms and Gender Order in the Long Nineteenth Century, sous la direction de I. Blom, K. Hagemann et C. Hall, Oxford, Berg, 2000, p. 111.

23. A. Oram : Women Teachers and Feminist Politics, 1900-1939, Manchester, Manchester University Press, 1996, pp. 101, 103, 132 ; D. Copelman : London's Women Teachers : Gender, Class and Feminism, 1870-1930, London, Routledge, 1996, pp. 25-26.

24. AHM, AR, 1913, p. 45.

25. A. Oram : Women Teachers..., op. cit., pp. 101, 103, 132 ; D. Copelman : London's Women Teachers..., op. cit., pp. 25-26.

26. A. MacKinnon : Love and Freedom..., op. cit., p. 133 ; M. Vicinus : Independent Women: Work and Community for Single Women, 1850-1920, London, Virago, 1985, p. 174 ; D. Copelman : London's Women Teachers..., op. cit., pp. 26-27.

27. M. Vicinus : Independent Women..., op. cit., p. 200.

28. Scheme for the Godolphin Ladies School... 25 April 1852, London, C. Roworth \& Sons, 1852 ; Scheme for the Management and Regulation of the Godolphin High School in the City of Salisbury... 6 May 1886..., Salisbury, Bennet Brothers, 1893.

29. M.A. Douglas, C.R. Ash: The Godolphin School..., op.cit., p. 78; Godolphin Magazine, $\mathrm{n}^{\circ}$ 21, 1902, p. 25 ; Anon. : Mary Alice Douglas, 1860-1914, Head Mistress of the Godolphin School 1890-1919, Wiltshire Record Office, s.d., p. 9 et p. 29.

30. AHM, AR, 1908, p. 8 et p. 78;1909, p. 6 ; CIL, Procès-verbal du 4 juillet 1912.

31. M.A. Douglas, C.R. Ash : The Godolphin School..., op. cit., p. 64.

32. T. Pakenham: The Boer War, London, Weidenfeld and Nicolson, 2000 (1979 pour la $1^{\mathrm{e}}$ éd.), p. 574.

33. M.A. Douglas, C.R.Ash: The Godolphin School..., op.cit., p. 56 et suiv.; J. Bush: Edwardian Ladies..., op. cit., p. 218 ; R. Strachey : Millicent Garrett Fawcett, London, John Murray, 1931, p. 198 et p. 205.

34. Salisbury and Winchester Journal, 29 mai $1909 ; 12$ novembre 1937.

35. M.A. Douglas, C.R. Ash : The Godolphin School..., op. cit., p. 202 et suiv.

36. Anon. : Mary Alice Douglas..., op. cit., pp. 7-9 ; Godolphin Magazine, $n^{\circ} 30,1905$, pp. 12-13 ; $n^{\circ} 55$, 1913, p. 26.

37. Godolphin Magazine, $\mathrm{n}^{\circ} 42,1911, \mathrm{p} .31$.

38. Ibid., $n^{\circ} 23,1902$, p. 38.

39. M. Douglas: The United Girls' Schools' Mission and Settlement. Letter from the Chairman of the Executive Committee, Godolphinarchive, s.d.

40. J. Bush : Edwardian Ladies..., op. cit., p. 141.

41. M.A. Douglas, C.R. Ash : The Godolphin School..., op. cit., pp.189-90 ; Salisbury and Winchester Journal, 29 mai 1909 ; Godolphin Magazine, n 58, 1914, p. 6.

42. Ibid., n² 21, 1902, p. 25.

43. Ibid., $\mathrm{n}^{\circ} 23,1902$, pp. 5-6.

44. M. Vicinus : Independent Women..., op. cit., p. 165.

45. M.A. Douglas, C.R. Ash : The Godolphin School..., op. cit., p. 171. 
46. R. Watts : "From Lady Teacher to Professional: a Case Study of Some of the First Headteachers of Girls' Secondary Schools in England », Educational Management and Administration, $\mathrm{n}^{\circ} 26$ (4), 1998, p. 348.

47. T. Pakenham : The Boer War..., op. cit., p. 580 ; Godolphin Magazine, ${ }^{\circ}$ 14, 1899.

48. Godolphin Magazine, $\mathrm{n}^{\circ} 14,1899, \mathrm{p} .20$.

49. Ibid., $n^{\circ} 15,1900$, p. $28 ; n^{\circ} 17,1901$, pp. $5-6 ; n^{\circ} 21,1902$, p. 15 ; M.A. Douglas, C.R. Ash : The Godolphin School..., op. cit., p. 187 et p. 189.

50. Godolphin Magazine, $\mathrm{n}^{\circ}$ 56, 1913, p. 9.

51. Official Report of the Pan Anglican Congress, London, SPCK, 1908, vol. 8, Section G, p. 25.

52. Godolphin Magazine, $\mathrm{n}^{\circ}$ 40, 1908, pp. 7, 19, 24, 20.

53. Ibid., n² 24, 1903, pp. 6-7.

54. B. Anderson : L'imaginaire national. Réflexions sur l'origine et l'essor du nationalisme, Paris, La Découverte, 1996.

55. Godolphin Magazine, $\mathrm{n}^{\circ}$ 24, 1903, p. 7.

56. Ibid., p. 12.

57. Ibid.

58. Ibid., $n^{\circ} 39,1908$, p. 7 ; $n^{\circ} 56,1913$, p. 13.

59. Ibid., $\mathrm{n}^{\circ} 47,1910$, p. $8 ; \mathrm{n}^{\circ} 53,1912$, p. 15.

60. Ibid., $\mathrm{n}^{\circ} 37,1907$, p. 9.

61. Ibid., n 49, 1911, p. $18 ; n^{\circ} 57,1914$, p. 26

62. Ibid., $\mathrm{n}^{\circ} 49,1911$, p. 18

63. I. Grewal : Home and Harem : Nation, Gender, Empire and the Cultures of Travel, Leicester, Leicester University Press, 1996, pp. 7-8.

64. Godolphin Magazine, $\mathrm{n}^{\circ} 39,1908$, p. 20.

65. Ibid., $\mathrm{n}^{\circ}$ 56, 1913, p. 14.

66. Ibid., n³3, 1906, p. 16.

67. J. Bush : Edwardian Ladies..., op. cit., chapitre 4.

68. Voir P. Chatterjee: The Nation and Its Fragments, Colonial and Postcolonial Histories, Princeton, Princeton University Press, p. 10 ; C. Hall : «Introduction : thinking the postcolonial, thinking the Empire ", in Cultures of Empire: Colonizers in Britain and the Empire in the Nineteenth and Twentieth Centuries. A Reader, sous la direction de C. Hall, Manchester, Manchester University Press, 2000, p. 13 et p. 15.

69. Godolphin Magazine, $\mathrm{n}^{\circ} 34,1906, \mathrm{p} .35$.

70. Ibid., n³9, 1908, p. 20.

71. Ibid.

72. Ibid., $\mathrm{n}^{\circ} 42$, 1909, p. 17. Voir P. Chatterjee : The Nation and Its Fragments..., op. cit., p. 10 ; C. Hall : «Introduction », in Cultures of Empire..., op. cit., p. 13 et p. 15.

73. Godolphin Magazine, $\mathrm{n}^{\circ}$ 42, 1909, p. 17.

74. Sur la « règle de la différence coloniale », voir P. Chatterjee : The Nation and Its Fragments..., op. cit., p. 10. Ce paragraphe (de même que la mise en forme du propos de cet article) s'inspire fortement de C. Hall: « The Rule of Difference », in Gendered Nations..., op. cit., pp. 108-112, ainsi que de C. Hall: « Introduction », in Cultures of Empire..., op. cit., p. 13 et p. 15.

75. Voir B. Anderson : L'imaginaire national..., op. cit.; C. Hall : «Missionary Stories : Gender and Ethnicity in England in the 1830s and 1840s ", in C. Hall : White, Male and Middle Class : Explorations in Feminism and History, London, Polity Press, 1996, p. 207.

76. I. Grewal : Home and Harem..., op. cit., p. 8 ; C. Kaplan, I. Grewal: «Transnational Feminist Cultural Studies: Beyond the Marxism/Postructuralism/Feminism Divides », Positions, Automne 1994, pp. 430-445. 
77. L. E. Nym Mayhall : «Introduction », in Women's Suffrage in the British Empire: Citizenship, Nation and Race, sous la direction de E.C. Fletcher, L.E. Nym Mayhall, P. Levine, London, Routledge, 2000, p. XII et p. XVI.

78. J. Goodman: "Their market value must be greater for the experience they had gained: secondary School headmistresses and Empire, 1897-1914 ", in Gender, Colonialism and Education: The Politics of Experience, sous la direction de J. Goodman, J. Martin, London, Woburn, à paraître, p. 181 et suiv.

79. Ce paragraphe se fonde sur P. Chatterjee: The Nation and Its Fragments..., op. cit., p. 10 ; C. Hall : "The Rule of Difference", in Gendered Nations..., op. cit., p. 110 ; C. Hall : «Introduction ", in Cultures of Empire..., op. cit., p. 20 ; A.L.Stoler, F. Cooper: «Between Metropole and Colony: Rethinking a Research Agenda ", in Tensions of Empire: Colonial Cultures in a Bourgeois World, sous la direction de A. L. Stoler, F. Cooper, Berkeley, University of California Press, 1997, pp. 3-4 et p. 7 ; M. Lake: "The Ambiguities for Feminists of National Belonging: Race and Gender in the Imagined Australian Community », in Gendered Nations..., op. cit., p. 172.

\section{RÉSUMÉS}

Sont examinés ici les liens entre les identités professionnelles, la religion, les missions, et l'Empire, qui ont déterminé le flux international d'enseignants et de directrices dans les écoles secondaires de filles entre la fin des années 1890 et 1914. Sont d'abord analysées les stratégies adoptées pour faciliter les migrations d'enseignantes du secondaire par l'Association des Directrices, organisation professionnelle basée en Angleterre. La seconde partie de l'article porte sur les missions religieuses et de la promotion de l'idée impériale, à l'échelon de l'école, à travers des études de cas provenant de la Godolphin School à Salisbury en Angleterre, et de deux écoles sud-africaines : St Michael à Bloemfontein, et l'école diocésaine de Grahamstown. La dernière partie de l'article met en évidence les processus d'identification culturelle au sein des trois écoles et conclut que celles-ci étaient impliquées, avec l'Association des Directrices, dans la construction de hiérarchies selon le sexe, la race, l'ethnie et la classe, à travers une complexe « règle de différence coloniale ».

This article explores inter-relations between professional identities, service, religion, mission, empire and Englishness that surrounded the international traffic of teachers and headmistresses in girls' secondary schools between the late 1890s and 1914. The article begins by examining strategies adopted to facilitate the overseas traffic of women secondary school teachers by the Association of Headmistresses, the key professional organisation in England for women teaching in girls' secondary schools. The second part of the article focuses on religious mission and mission to empire at the level of the school using case study material from Godolphin School, Salisbury, England, and two South African schools, St Michael's Bloemfontein, and the Diocesan School, Grahamstown. The last section of the article points to layers of cultural belonging at the level of the three schools and concludes that the three schools and the Association of Headmistresses were implicated in constructing hierarchies along the axes of gender, race, ethnicity and class through a complex « rule of colonial difference ».

In pointierter Weise zeichnet der Beitrag die Zusammenhänge zwischen Berufsbildern, Religion, Mission und Empire nach, die den internationalen Zufluss von Lehrerinnen und Direktorinnen in 
den Mädchenoberschulen zwischen den ausgehenden 1890er Jahren und 1914 bestimmten. Dabei wird zunächst untersucht, mit welchen Mitteln der Direktorinnenverein, eine Berufsvereinigung mit Sitz in England, die Versetzungsbereitschaft von Lehrerinnen zu fördern versuchte. Der zweite Teil des Beitrags beschäftigt sich mit den religiösen Aufgaben und der Vermittlung der Idee vom Empire auf der Ebene der Schule anhand von Fallstudien aus der Godolphin School in Salisbury in England und aus zwei südafrikanischen Schulen, St. Michael in Bloemfontein und die Diözesanschule in Grahamstown. Im letzten Teil des Beitrags werden schließlich die kulturellen Identifizierungsprozesse in den drei Schulen herausgestellt; es ergibt sich, dass in diesen Schulen über den Direktorinnenverein eine Hierarchisierung nach Geschlecht, Rasse, Volkszugehörigkeit und Schicht erreicht wurde und somit ein komplexes Regelsystem, « kolonialer Differenzierung » aufgebaut wurde.

INDEX

Index géographique : Empire britannique, Royaume-Uni, Afrique du sud

Mots-clés : colonie, enseignante, enseignement secondaire, histoire du genre, identité professionnelle, mission

Index chronologique : XIXe siècle, XXe siècle

\section{AUTEURS \\ JOYCE GOODMAN}

King Alfred's College, Winchester 